\title{
Requirements Gathering for the Delivery of Healthcare Data in Aware Homes
}

\author{
Julie Doyle, Brian O’Mullane, Ann O’Hanlon*, R. Benjamin Knapp \\ CASALA, Netwell Centre* \\ Dundalk Institute of Technology \\ Co. Louth, Ireland \\ julie.doyle@casala.ie
}

\begin{abstract}
Aware homes support the monitoring of older adults, with the potential to detect a wealth of information regarding the person's functional, cognitive and social wellbeing. While much research in this space focuses on the collection and interpretation of sensor data, it is equally important to understand how we can begin to relay the information learned back to older adults, empowering them to play an active role in the management of their health. In this paper we discuss the results of the requirements gathering phase of our research with a number of older adults living in aware homes. The goal of this research is to explore issues surrounding the delivery of healthcare information, as collected through embedded sensors in the home, to the home owner. Our results reveal a number of emerging themes around this space, including a desire by older adults to play an active role in managing their health and potential concerns surrounding the delivery of such information through technology.
\end{abstract}

\section{INTRODUCTION}

It is a well-known and often discussed fact that the world's population is ageing. By 2050 an estimated 22\% of people (almost 2 billion) will be aged 60 or over [16]. This will result in a smaller workforce and therefore a shortage of available health care workers to look after the older population. Thus, there is an increasing realisation that with an ageing population comes a pressing need to support independent living and to help older adults to self-manage their health, in the place of their choice [1], [11]. Research on improving the quality of life of older adults is being aided by the proliferation of low-cost wireless sensor systems and healthcare ICT (Information and Communication Technologies), both of which are becoming increasingly pervasive, moving from controlled and clinical environments into real homes [6]. Assisted living healthcare technologies have the potential to enable older adults to stay active and productive for longer, to engage in society and to enjoy a healthier quality of life for longer. Such technologies include wearable and embedded sensors to monitor health [5]; alarm systems that trigger an event, for example when a person has suffered a fall [15] and interactive ICT that encourage older adults to maintain and/or enhance physical, social and cognitive activity [8], [12].

As a consequence of such technologies moving into homes, new challenges arise in working with end users of these systems to ensure that they are not only usable, but that the technologies in place are of benefit to the user, thus encouraging their usage. We are particularly interested in embedded sensor systems within Ambient Assisted Living (AAL) research and how to relay health-related data collected from multiple sensors back to the older adult. While research on sensor systems and detecting patterns of behaviour dominates the AAL research space, there is much less research on what should be done with this collected data, and this has motivated our research. Novel methods are needed to deliver health data back to older adults, to make them aware of their health, to ensure they understand the data and what to do with it and to allow them to decide who they want to share it with. It is also necessary to understand any concerns older adults have about receiving such information.

Ultimately, our research goal is to close the loop in AAL systems, not only monitoring and tracking older adults in their homes, but also supporting interventions that will allow them to play a greater role in actively managing their health and support them in changing behaviours to improve their wellbeing. This will help older adults to live independently in the place of their choice for longer. In this paper we present findings from semi-structured interviews conducted with nine older adults who are residents of aware homes. The aim of the interviews was to explore attitudes, opinions and concerns that older adults have about actively managing their health care. Our findings indicate that while older adults are very accepting towards receiving data on their health, there are a number of concerns that need to be addressed in the design of interactive healthcare technologies.

\section{BACKGROUND - THE GREAT NORTHERN HAVEN}

Our research involves working with a number of older adults living at the Great Northern Haven (GNH), a demonstration housing project consisting of 16 purpose-built homes, each equipped with a combination of sensor and interactive technology to support AAL for older people. There are a total of 2240 sensors and actuators throughout the development, with approximately 140 sensors in each home. Currently there are 13 homes occupied by 11 men and 4 women. To date, we have collected a vast amount of data from the embedded sensors within each of these homes and models are being built to detect patterns in activities of daily living and health. The sensors include PIR sensors to detect motion; a number of window and door sensors to detect and report open and closed events and sensors to detect power consumption and 
heating usage. In addition each home has a number of alarm cords and buttons, a home security system and a telecare device that links with a monitoring service.

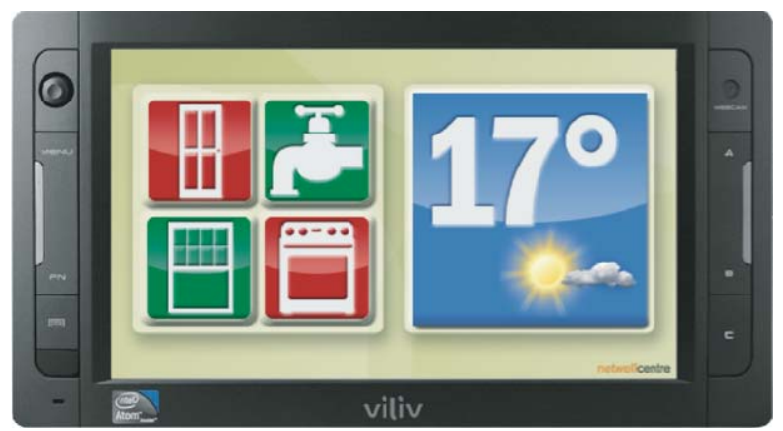

Figure 1. ALAN - Assisted Living Access Network

Given the large amount of data we have collected, and are continuing to collect, there is the potential to infer and convey a wealth of information to the resident, including information specific to their health. For example, detecting changes in speed of movement through the home might suggest declining mobility. Unusual flow through the home might be indicative of cognitive decline. In addition to cognitive and physical function, we plan to detect changes in social behaviour and mood. Our technology interventions will aim to address such changes in behaviour, making the resident aware and ideally supporting them in changing or adapting to this behaviour. It should be noted that the homes support the deployment of home automation rules that may be used for interventions, for example turning on lights when a particular event is detected. Furthermore, a comprehensive wiring system is in place to allow further home automation and healthcare devices to be added.

To deliver feedback gathered from the home to residents we have installed two interactive devices into each of the homes. These are an Internet-ready television we call STEVE (Senior Television Enhanced Viewing Experience) and a touch sensitive tablet device we call ALAN (Assisted Living Access Network). The aim of both devices is to deliver services to older adults through a series of applications, which can include anything from news services, cognitive games and local services to the delivery of health related information. Current applications on ALAN include a weather application and a home monitoring application. For example if a door is opened or the stove is on, the corresponding icon is coloured red (Figure 1). The aim of the research outlined in this paper is to engage with the residents of GNH to understand their attitudes and concerns about receiving information relating to their health, consequently aiding in the design of health care applications for STEVE and ALAN.

\section{RELATED WORK}

There is a vast amount of ongoing research within the aware home for AAL space, with past and current projects including initiatives at the ORCATECH living laboratory in Portland, Oregon [10] and the Enable project, with partners situated in five European countries [9]. The majority of such research focuses on how best to monitor older adults and their environments to detect changes, and possibly decline, in a person's bio-psycho-social wellbeing. Comparatively little research examines issues surrounding relaying the information gathered back to interested stakeholders so that interventions can be put in place to enhance an older adult's health. Those that do discuss interventions talk about doing so to provide information to caregivers and clinicians on when to intervene [14]. Typically, the person being monitored is not given control over their health data. However researchers are beginning to address this issue.

A study presented in [13] involves monitoring how well older adults perform activities of daily living (ADLs) and presenting this information back to older people, their caregivers and clinicians. The study probed older adults on whether they would find various health care information useful, including a monitor for their medication and information on activity performance including making a cup of coffee and telephone usage. The older adults they interviewed $(\mathrm{N}=4)$ stated that they would be interested in this information as they got older, but also that they would want to know why there were declines in ADLs so that they could 'fix' any problems [13]. A study by Bhachu et al. focused on how older adults felt about technology devices that might deliver health-related information, rather than attitudes to the type of data they might display [4]. The study introduced a focus group of older adults to various stand-alone technology devices, exploring their opinions on each device and its utility, in addition to how they would feel about viewing their health statistics on such devices. The majority of participants were positive about receiving such information. The potential barriers of technology were seen as trivial when compared to health benefits. Despite this, there was concern regarding the stigma attached to stand-alone technology displaying private health data in ones' home. Privacy is another concern often discussed in the literature. For example, one study found that people who suffer from a disability are more open to sharing data about their health than those without a disability [3]. In the study by Lee and Dey [13], older adults reported having no issues about sharing health information with their family members, friends, or clinicians. While this is positive, it is important that older adults are given control over who has access to their data. Cantor suggests a policy of 'No information about me, without me' to ensure older adults make their own choices regarding home monitoring and protection of their privacy and autonomy [7].

\section{METHODOLOGY}

To gather data on how the residents of GNH felt about receiving feedback regarding their health, we conducted oneon-one semi-structured interviews with the residents. We spoke with 9 of the residents (6M, 3F, age range 60-79), as some were unavailable due to illness or travel. Interviews lasted from 20-30 minutes. All data were audio recorded and transcribed for later analysis. Two of the 9 residents interviewed (R5, M; $\mathrm{R} 16, \mathrm{~F})$ own a PC or laptop and use them regularly. Both residents reported that they heavily rely on Internet services for many activities, such as transport timetables, directions and internet banking. Both residents also have a Facebook page and Skype accounts. 
As the goal of the interviews was to understand the attitudes of residents towards receiving information on their health, the categories of questions asked included:

- What health related services they currently access and how.

- What (if any) healthcare-related technology they currently use at home.

- What type of health services they would like to access from their home.

- Their interest in receiving feedback on their health status, as gathered through sensors in their home.

- Any potential concerns regarding receiving such data.

We also used sample diagrams, such as charts and graphs, to indicate how such data might be presented to residents through either ALAN or STEVE. A discussion around our results is presented in the following section.

\section{DISCUSSION}

While the benefits of monitoring are evident, there are a number of reasons why residents might express concern over such systems and particularly on receiving feedback on their health. Declining functional or cognitive ability can be perceived as a person becoming older, weaker and ultimately more dependent. There might be concerns over privacy of data and who has access to sensitive health information. As Cantor notes, hiding or not discussing one's health problems is not possible when a monitoring system has been installed [7]. Each GNH resident was aware of the embedded sensors within their home, and the purpose of them, prior to moving in. As such, a level of acceptance around monitoring was expected. However, at the start of the interview process, a conversation took place with the resident to review exactly the type of information and services we could potentially provide to them. All residents were very forthcoming regarding their health and their concerns for their health as they got older. There was a general feeling that any 'intervention' focused on improving their quality of health and wellbeing could only be a good thing. While the purpose of the sensors is well understood, there is still an uncertainty as to how technology can deliver such data and more so, that they themselves will be capable of using the technology. The most important themes that emerged are discussed in the following subsections.

\section{A. Self-management of one's healthcare}

Quantitative and qualitative assessments conducted with residents prior to these interviews taking place, reveal a large range of illnesses including diabetes, heart problems, chronic back pain, depression, anxiety and cognitive decline. All of the residents we interviewed felt that their health was worse now than it was a year ago. Despite this, 6 of the 9 residents were hopeful that their health would improve in the following year. There was a level of expectation that the aware homes would provide residents with a better quality of life. Probably our most interesting and promising finding is that all residents expressed an interest in receiving health related data and being involved in their health management. Already, the two residents who own a $\mathrm{PC}$ or laptop play an active role in the management of their health, or as in R16's case, their spouse's health. R16 closely monitors her husband's medication prescription. When he is prescribed something new, she reported routinely checking that the new medication does not interfere with his existing heart medication. R16 said: "She (the doctor) knows about his heart and isn't going to give him anything that could affect him, but I still check. I have to check". She recently requested that the doctor reduce the strength of a sleeping pill that had been prescribed to her husband as it made him groggy during the day. R16 realised, through an Internet search, that a lower dosage was available. R17 suffers from back pain and said he would like to keep a diary of when his back is bothering him, when it causes him to have trouble sleeping and possible reasons for this based on his routine on a particular day. This way, he would have a 'report' when he went to visit his GP. However, while he had begun to do this he said it was difficult to keep it up as most days he forgets.

R5 reported using his GP as the first point of contact in accessing information on his health. However, following a doctor's visit he will visit medical websites to learn more about his condition and to understand what medication he has been prescribed. He clarified: "I'm not saying self-diagnosis, I'm not suggesting that at all. But after you've seen the doctor then it's $o k$ ". Many other residents were involved in some level of management of their health, such as maintaining a healthy diet, taking blood pressure or remembering to take medication. The most common medical device used by residents is a blood pressure monitor, with one resident keeping a paper record of his blood pressure. Those residents who do not currently have Internet access discussed how it might help their doctor if they could bring them a 'print-out' of the information learned from the sensors.

Self-management of chronic health conditions has been described as an individual's ability to manage the symptoms, treatment, physical and psychological consequences and life style changes inherent in a health condition [2]. Given the interest expressed in taking control of one's health, we have decided to include a daily health survey to enable residents to keep a record of their day to day wellbeing and mood and to detect patterns of change in these that might contribute to depression or ill health. Furthermore, we plan to integrate blood pressure and weight readings into the health survey, through wireless devices that can interact with STEVE and ALAN. The benefit of a daily survey that includes feedback on the resident's perception of their wellness is that it can provide a ground truth measure to accompany the sensor data, providing a direct insight from the resident as to why changes are occurring. As highlighted by Lee \& Dey [13], the 'Why' is necessary when delivering health related data, to help stakeholders to interpret it and to decide on an intervention to address it.

\section{B. Residents' concerns}

Privacy was the main concern regarding receiving and storing health related data through technology in the home. Specific concerns were raised by three residents about the 
possibility of someone breaking into their home and stealing their TV or tablet device. Despite the fact that residents were living with monitoring technology, only two residents expressed concern over privacy issues surrounding this, with R5 referring to it as a little like 'Big Brother'. R8, the oldest of those we interviewed (age $=79$ ) was concerned about what researchers would be able to see her data. She also wanted clarification that the sensors are not cameras. While this had been explained to residents prior to moving in, providing a paper booklet of how the data from the sensors appears would help put residents' minds at ease. It is critical that the resident is aware of who is likely to have access to their data, and that they retain control of this.

There were also concerns raised about the ability to interpret health data and the need to get help with this. One resident talked about asking his doctor for help on what to do with the data. He noted: "You have to make an appointment to go to the doctor though. You can't just go down. If you told me there's a (health) problem it might worry me". The majority of residents $(\mathrm{N}=7)$ were concerned about using technology, having never used a computer before. One resident commented "Sure how would you even turn it on". It is vital to ensure that technology does not act as a barrier to older adults playing an active role in the management of their health. A benefit of delivering healthcare information through technologies such as ALAN or STEVE is that they are not computers and thus are potentially more acceptable to older adults.

\section{Considerations for researchers}

Before technologies to deliver health information to older adults can be designed, it is necessary to understand the attitudes of older people towards receiving and acting on health information. What was interesting was that the majority of residents expressed strong motivation to receive health related data, even the younger residents who felt that it was not immediately necessary: "It mightn't be such a benefit now... but it will be in time". They perceived a sense of security in prevention rather than cure. It was also noted that it would be best to learn how to use the technology before it became a necessity. The most cited health related concern residents had about ageing was becoming forgetful, with many residents already feeling absent minded. As such, when asked about potential health services including physical games, social technology or cognitive 'brain' games, the latter was most popular as residents feel they have the least control over their memory and are unsure of how they can try to 'prevent' decline.

Technology design to support health self-management should integrate elements of self-efficacy, goal setting, social support and motivation. Not only should older adults feel competent in managing their health, but the technology should be engaging, encouraging them to take an active role. It is also necessary to educate older people on the benefits of long term monitoring of health. One resident noted: "I wouldn't have to go on the TV (Internet) to know that I wasn't well". While this is very true, we need to help older people to understand that embedded sensors can detect very subtle changes that they themselves may not be aware of. Thus we plan to conduct a series of classes around the sensors and the health issues that can be detected through monitoring, using visualisations of examples and allowing residents to interact with the healthcare services on STEVE and ALAN before we expect them to start using them in their homes.

\section{ACKNOWLEDGMENT}

The Centre for Affective Solutions for Ambient Living Awareness (CASALA) is an applied research centre on the Dundalk Institute of Technology (DkIT) campus. CASALA has been established and initially funded under the Enterprise Ireland Applied Research Enhancement (ARE) programme, which aims to build applied expertise within the Institutes of Technology and makes this knowledge accessable to local and national industry. www.casala.ie

\section{REFERENCES}

[1] N. Alm, P. Gregor and A.F. Newell. Older people and information technology are ideal partners. In International Conference for Universal Design, (2002).

[2] I J. Barlow, C. Wright, J. Sheasby, A. Turner and J. Hainsworth. Selfmanagement approaches for people with chronic conditions: A review. In Patient Education and Counselling, 48, (2002), pp. 177-187.

[3] S. Beach, R. Schulz, J. Downs, J. Matthews, B. Barron and K. Seelman. Disability, age and informational privacy attitudes in quality of life technology applications: Results from a national web survey. In ACM Transactions on Accessible Computing, 2, 1, (2009).

[4] A.S. Bhachu, N.A.Hine, J.L. Arnott. Technology devices for older adults to aid self management of chronic health conditions. In 10th ACM SIGACCESS Conference on Computers and Accessibility (ASSETS), ACM, (2008).

[5] V. Bianchi, F. Grossi, G. Matrella, I. De Munari, and P Ciampolini. A wireless sensor platform for assistive technology applications. In 11th EuroMicro Conference on Digital Systems Design Architectures, Methods and Tools, (2008), pp. 809-816.

[6] P.Bonato. Advances in Wearable Technology and Applications in Physical Medicine and Rehabilitation. In Journal of NeuroEngineering and Rehabilitation, 2, 2, (2005).

[7] M.D. Cantor. No information about me without me: Technology, privacy and home monitoring. In Generations, 30, 2, (2006), pp. 49-53.

[8] J. Doyle, C. Bailey, B. Dromey and C. Ni Scanaill. BASE - An Interactive technology solution to deliver balance and strength exercises to older adults. In Pervasive Health, IEEE, (2010).

[9] The Enable Project. Available at: http://www.enableproject.org/index.html (accessed January 2010).

[10] T.L. Hayes, T. Riley, M. Pavel, J.A. Kaye. Estimation of rest-activity patterns using motion sensors. In IEEE Engineering and Medicine and Biology Society, (2010).

[11] C. Jones., C. Winegarden and W. Rogers. Supporting healthy aging with new technologies. In ACM Interactions, 16 (4), (2009), pp. 48-51.

[12] D. Kern, M. Stringer, G. Fitzpatrick, and A. Schmidt. Curball - a prototype tangible game for intergenerational play. In 15th IEEE International Workshop on Enabling Technologies: Infrastructures for Collaborative Enterprises, (2006), pp. 412-418.

[13] M.L. Lee and A.K.Dey. Embedded assessment of aging adults - A concept validation with stakeholders. In Pervasive Health, IEEE, (2010).

[14] M. Pavel, H. Jimison, T. Hayes and J. Kaye. Technology in support of successful aging. In lemph\{National Academy of Engineering - Bridge Issue on Technologies for an Aging Population, 39, 1\}, (2009).

[15] A. Sixsmith and N. Johnson. A smart sensor to detect the falls of the elderly. In IEEE Pervasive Computing, 3(2), (2004).

[16] United Nations, World Population Ageing, 2007. 